\title{
Productivity vs. Production Capacity: Hospitalists as Medical Educators
}

Jeff Wiese, MD, FACP, FHM
Department of Medicine, Tulane University Health Sciences Center, New Orleans, Louisiana.

Disclosure: Nothing to report.
Maintaining a balance between productivity (what we do now), and production capacity (the ability to continue to do what we now do in the future) is one of the central tenets of successful organizations and societies. The economic benefits of industrialization (production) must be balanced with the needs of the environment (production capacity). It is appropriate then, that this issue of The Journal of Hospital Medicine juxtaposes Conway's ${ }^{1}$ article on hospitalists' current "production" of ensuring value, with articles addressing medical education, our production capacity.

What is the role of hospitalists in medical education? The article by Beasley et al., ${ }^{2}$ confirms what has long been suspected: the growth of hospitalists in medical education has paralleled the growth of the movement as a whole. The meta-analysis by Natarajan et al. ${ }^{3}$ further suggests that hospitalists are at the very least no worse in medical education than other specialists, and in some domains, may be superior. The theoretical fears of hospitalists as medical educators have not been borne out: utilizing hospitalists as educators does not lead to a decline in resident autonomy, nor does it lead to a decline in educational ability. But despite the fact that $73 \%$ of residency training programs utilize hospitalists, there are several reasons why the hospitalists' role in inpatient medical education should be even more robust.

The landscape of graduate medical education has dramatically changed in the past 10 years. The knowledge-only paradigm has evolved to a comprehensive focus on the trainee's overall performance, including understanding the healthcare system in which she works (systems of care), self-reflection on her practice (practice-based learning), and an augmented emphasis on professionalism and interpersonal skills. ${ }^{4}$ Unfortunately, many systems have not undergone a similar paradigm change, opting instead to merely rearrange components of the old knowledge-focused system. For example, practicebased learning may remain relegated to journal clubs, where the focus is on the knowledge contained in the chosen article, instead of active exercises in which the resident self-reflects on his patient care performance and seeks ways to improve that performance. Instruction in systems-of-care may remain within a knowledge-only paradigm: a didactic lecture on Medicare/Medicaid and reimbursement instead of residents actively participating in quality improvement projects.

The article by Mazotti et al., ${ }^{5}$ provides insight into the reason for this developmental arrest: “there isn't enough time in the old system." The duty-hours have decreased for residents, but since the work product has remained the same, the result has been an increase in work intensity. As work intensity increases, production capacity (medical education) is the first to be sacrificed in an effort to maintain production (getting the work done).

Here is the yet unrealized role of the hospitalist. The same degree of systems reengineering that brought about improved efficiency in patient care (shorter lengths of stay, fewer readmissions), must be applied to the inpatient education system if duty hours, a reasonable work intensity, and meaningful education in each of the core competencies are to coexist. But there is no panacea for these issues: each system is unique, and the solutions to improving the efficiency of the inpatient education environment are just as unique. Solutions require a "systems architect" (ie, the hospitalist) to redesign the educational environment in his particular system. It is natural to assume that the hospitalist educator, familiar with the strengths and weakness of the educational system in which he routinely works, will be best equipped to enact meaningful solutions that improve efficiency while protecting the principles of medical education.

What does it mean to "change the educational system?" Taiichi Ohno, ${ }^{6}$ Toyota's Chief Engineer, provides "Seven Organizational Wastes," a framework for identifying areas of improvement in the educational work environment. Consider, for example, the following selected opportunities for improving the efficiency of the inpatient medical education system: (1) excessive testing or consultation leading to delayed discharge and more resident work effort (Overproduction); (2) the resident team waiting for the attending to arrive from a procedure or clinic (Waiting); (3) inadequate teaching about the principles of transitions of care, resulting in more readmissions to the teaching service (Transporting); (4) failure to have quality improvement conferences to discuss the appropriateness of admissions (Inappropriate processing); (5) failure to teach residents how to work with social work/placement services to facilitate early discharge (Unnecessary inventory); (6) failure to construct a training program that limits fragmentation, with residents moving from 1 task to the next and back again (Unnecessary motion); and (7) medical errors resulting in prolonged lengths of stay and resident work effort (Defects). There is no shortage of opportunities for improving the efficiency of 
the inpatient educational environment, but it requires that the systems architect is sufficiently familiar with the system to design interventions that are meaningful and effective. This is the unique advantage of the hospitalist.

But the greatest risk to inpatient medical education is yet to come, and it may be on the hospitalist's shoulders to reverse a dangerous trend. With the advent of more extensive electronic medical records, the locus of patient care has begun to shift from the bedside to a computer terminal. An unbridled drive to efficiency, without a steward to ensure the primacy of patient-centered care, is likely to inspire the next generation of physicians to see "Mr. A. Huxley" as the "i-Patient," in which the entirety of his management is conducted from the safety of a computer terminal. ${ }^{7}$ Despite the need for efficiency, the patient has to remain the focal point. It is at the bedside that the resident learns that observing 5 bags of potato chips on the nightstand might obviate a million-dollar workup for refractory hypertension. And it is at the bedside that the resident learns that despite our elaborate protocols and decision analyses, the ultimate testing and management decisions hinge upon the patient's preferences. The single best thing than can be done to augment patient safety and quality is to maximize the time the patient spends with his healthcare team; and the hospitalist, who is not in a rush to complete morning rounds in an effort to get to a clinic or an endoscopy suite, may be the person who has the time to prioritize bedside rounds as a part of the educational environment.

The article by Nazario $^{8}$ establishes the urgency of integrating the humanities into patient management. For meaningful humanities instruction to occur, however, it has to occur at the bedside, not in the classroom. And this requires that the supervising physician has the time to reflect with the resident upon the humanism issues that are unique to each patient encounter. Once again, it is time that enables the luxury of this self-reflection, a commodity that the hospitalist enjoys as a part of her job description. It is also a commodity that the hospitalist can generate by augmenting efficiency in the educational system, provided patientcentered care remains the priority.

The role of the hospitalist has to be much more than merely patching the current paradigm of graduate medical education. Overseeing nonteaching services and serving as the night float physician are examples of these patches. While valuable, these roles are useful only in preserving production of the current system, not in enabling production capacity for the next generation of physicians. Continuing in this role without also becoming an active part of the teaching service sends a message to residents that a career as a hospitalist in a teaching environment is only to be a fourth-year or fifth-year resident. Why would any resident embark upon that career? Meeting the demand for 30,000 hospitalists by 2012 requires a pipeline of physicians, and answering this question will be central to achieving that goal. Residents must have hospitalist role models, occupying careers devoted to patient safety and quality; careers that are meaningful and fulfilling.
To this end, the hospital medicine community cannot be satisfied with being "no worse than other specialists in medical education." As Natarajan et al. ${ }^{3}$ point out, the evaluation of inpatient medical education has, with few exceptions, been solely based upon learner's subjective opinions. Meaningful change in the educational system will require meaningful objective endpoints: participation in the quality improvement projects; patient-centered evaluations of resident performance; end-user evaluations of resident communication skills (clinic physicians, nurses, other services); metrics to assess the efficacy of the transition of care; and resident profiles that enable self-assessment of their practice. It will be up to the hospitalist to assess the system to define these meaningful endpoints that ensure that inpatient education is advancing quality, safety, and patient-centered care.

Despite all of the reasons for why hospitalists should be more involved in the teaching service, there remains the 1 reason that they are not: hospitalists on average are young, and they may not have the teaching skills that more experienced generalists or subspecialists possess. To bring about the benefits hospitalists can offer to the inpatient education environment, hospitalists must be willing to compensate for their lack of teaching experience by seeking out formal training courses in medical education. The Academic Hospitalist Academy cosponsored by the Society of Hospital Medicine (SHM), the Society of General Internal Medicine (SGIM), and the Association of Chiefs of General Internal Medicine (ACGIM) is 1 example. $^{9}$

As experience is accumulated, more hospitalists have to actively seek out leadership positions in graduate medical education, aligning our strengths, in patient safety, efficiency, and systems change, with the goals and objectives of the residency and student programs. The fact that $73 \%$ of residency training programs utilize hospitalists is exciting; the fact that there are only 15 hospitalists as program directors is disappointing. ${ }^{2}$ As was the case in the clinical care environment, leadership will be just as important in the education environment, as it is important for enacting the changes that will transform the inpatient education environment to a system that is efficient, safe, and patient-centered.

Hospitalists have been effective in their production, augmenting efficiency and quality of patient care. But the reality is that the task of ensuring value, as it pertains to patient safety and quality, is too onerous of a task to be accomplished with arithmetic gains. Generations of physicians will have to adopt a cultural change to reach the ultimate goal. It is of little consequence that we improve safety for a moment in time, only to have it fall by the wayside as successive generations of physicians take our place. Now is the time for hospitalists to fully embrace the inpatient education environment as our responsibility. Too much time has already been wasted in arguing against duty-hours regulations. These regulations are now here to stay, and this has created a system that is currently unable to handle the strains of multiple demands. Only by using the hospitalist's expertise in systems improvements and efficiency will a new 
inpatient educational system evolve; one that is efficient, safe, and patient-focused, thus ensuring current production. And also one that enables meaningful development of communication, practice-based learning, and systems-of-care skills, ensuring our production capacity for years to come.

\section{Address for correspondence and reprint requests:}

Jeff Wiese, MD, FACP, FHM, 1430 Tulane Ave., New Orleans,

LA 70112; Telephone: 504-988-1143; Fax: 504-988-3971;

E-mail: jwiese@tulane.edu

Received 8 July 2009; accepted 3 August 2009.

\section{References}

1. Conway PH. Value-driven health care: implications for hospitals and hospitalists. J Hosp Med. 2009;4(8):507-511.

2. Beasley BW, McBride J, McDonald FS. Hospitalists involvement in internal medicine residencies. J Hosp Med. 2009;4(8):471-475.
3. Natarajan P, Ranji S, Auerbach A, Hauer K. Effect of hospitalist attending physicians on trainee educational experiences: a systematic review. $J$ Hosp Med. 2009;4(8):490-498.

4. Accreditation Council for Graduate Medical Education (ACGME). Internal Medicine Program Requirements. Available at: http://www.acgme.org/ acWebsite/RRC_140/140_prIndex.asp. Accessed August 2009.

5. Mazotti LA, Vidyarthi AR, Wachter RM, Auerbach AD, Katz PP. Impact of duty hour restriction on resident inpatient teaching. J Hosp Med. 2009; 4(8):476-480.

6. Ohno T. Just-In-Time for Today and Tomorrow. New York, NY: Productivity Press; 1988.

7. Verghese A. Culture shock: patient as icon, icon as patient. $N$ Engl J Med. 2008;359:2748-2751.

8. Nazario R. The Medical humanities as tools for the teaching of patientcentered care. J Hosp Med. 2009;4(8):512-514.

9. Society of General Internal Medicine (SGIM). The Academic Hospitalist Academy. Available at: http://www.sgim.org/index.cfm?pageId=815. Accessed August 2009. 